\title{
Tumor Calcification: A New Response Pattern of Myxoid Liposarcoma to Trabectedin
}

\author{
Anthony Turpin ${ }^{a} \quad$ Sophie Taieb ${ }^{b} \quad$ Nicolas Penel $^{a}$ \\ ${ }^{\mathrm{a}}$ Department of Medical Oncology, and ${ }^{\mathrm{b}}$ Imaging Department, Centre Oscar Lambret, \\ Lille, France
}

\section{Key Words}

Myxoid liposarcoma $\cdot$ Trabectedin $\cdot$ Tumor calcification

\begin{abstract}
Introduction: Myxoid/round-cell liposarcoma (MRCL) is a specific histological subtype that accounts for $30-35 \%$ of liposarcomas and whose virulence depends on the quantity of round-cells within the tumor. $\mathrm{MRCL}$ is associated with specific chromosomal translocations resulting in the formation of CHOP/FUS and CHOP/EWS fusion proteins. A high sensitivity of MRCL to trabectedin was reported. Case Report: We report the case of a 63-year-old woman with a bulky and metastatic $M R C L$, treated with trabectedin $1.5 \mathrm{mg} / \mathrm{m}^{2}$ as a first-line treatment. She experienced a long-lasting clinical benefit. The patients received 14 cycles of trabectedin and achieved a durable partial response to the metastases and a stable disease of the primary tumor, which is a very favorable safety profile. Also noteworthy is that we have observed a calcification of the primary tumor and the metastasis. The response, which lasted 30 months, led to a symptomatic improvement, associated with an excellent general condition and an absence of pain. Conclusion: To the best of our knowledge, this is the first report of a MRCL treated with trabectedin that resulted in a calcification of the primary tumor and the metastases, associated with an outstandingly long response. This case suggests that trabectedin may represent a feasible first-line therapeutic option for patients with $M R C L$, with meaningful clinical benefits and an acceptable safety profile.

(C) 2014 S. Karger AG, Basel
\end{abstract}

\section{Background}

Myxoid/round cell liposarcoma (MRCL) is a specific histological type within the family of adult soft tissue sarcomas (STS) that accounts for one-third of liposarcomas and $10 \%$ of 
all adult STS [1]. MRCL is a translocation-related liposarcoma associated with specific chromosomal translocations. It involves chromosome 12 and, more precisely, the transcription factor CHOP, mainly $\mathrm{t}(12 ; 16)(\mathrm{q} 13 ; 11)$, with the CHOP/FUS genes or the rarer $\mathrm{t}(12 ; 22)(\mathrm{q} 13 ; \mathrm{q} 12)$ chromosomal translocations involving the CHOP/EWS genes [2, 3]. The resulting formation of fusion proteins CHOP/FUS and CHOP/EWS act as constantly activated and deregulated transcriptional factors and therefore stimulate cell proliferation. Although therapeutic options for this subgroup of tumors are limited, patients with MRCL are highly responsive to trabectedin, which proofed to have anti-proliferative effects on MRCL cell lines at low nanomolar concentrations [4]. The high response rates achieved with trabectedin in MRCL, marked by early radiological alterations in tumor tissue, often precede delayed tumor shrinkage and suggesting a selective mechanism of action for this translocation-related sarcoma. Recent data suggest that trabectedin interferes in the manner of a targeted therapy with the transcriptional activity of the fusion gene in MRCL $[1,5]$.

In 2007, trabectedin (Yondelis ${ }^{\circledR}$, PharmaMar) was the first marine-derived antineoplastic drug approved by the European Medicines Agency for the treatment of patients with advanced STS after anthracyclines and ifosfamide failure and who are unsuited to receive these agents. Trabectedin has a unique mechanism of action. It is based on the interaction with the minor groove of the DNA double helix and it affects gene transcription and DNA repair pathways, resulting in G2-M cell cycle arrest and ultimately apoptosis [6]. Trabectedin shows nearly similar response rates in leiomyosarcoma and liposarcomas (Lsarcomas) as those in combination therapies and is less toxic by far [7]. Nevertheless, the greatest advantage of trabectedin has been observed in patients with advanced myxoid liposarcoma $[3,8]$.

We hereby report the case of a 63-year-old, heavily pre-treated woman with bulky and metastatic MRCL who achieved a prolonged disease remission following treatment with trabectedin. The rarity of calcification of the primary tumor, the metastases as well as an outstandingly long response to trabectedin prompted this case report.

\section{Case Presentation}

A 63-year-old woman without any underlying disease was diagnosed with a large tumor in the posterior part of the left thigh in January 2011. The first magnetic resonance imaging showed a heterogeneous mass with a diameter of $295 \mathrm{~mm}$ (fig. 1a). The patient was initially managed by a surgeon at a tertiary center. The community center surgeon performed an open biopsy, which resulted in a wound that never healed. The diagnosis was myxoid liposarcoma associated with small round cells (MRCL). The chest and abdominopelvic CT scan revealed the presence of multiple metastases, including a mediastinal mass (38 $\mathrm{mm}$; fig. 1c).

Based on the metastatic spread and the nature of the disease, the multidisciplinary committee suggested the inclusion of the patient into a randomized, multicenter phase III study (ET-C-002-07 study; ClinicalTrials.gov identifier: NCT00796120) that evaluated trabectedin $1.5 \mathrm{mg} / \mathrm{m}^{2}$ given as a 24-hour intravenous infusion $\mathrm{q} 3 \mathrm{w}$ versus doxorubicinbased chemotherapy as a first-line therapy in patients with translocation-related sarcomas. Once the patient signed the informed consent form, she was randomly assigned to the trabectedin arm. Before chemotherapy, the tumor was painful. That resulted in the administration of opioids and it was associated with severe asthenia. The first cycle of treatment was administered in March 2011. The patient received 14 cycles until January 2012. The treatment was then stopped due to hematological toxicity (grade 3 anemia, grade 
3 thrombopenia, and recurrent infections). Throughout the trial, the tumor response was evaluated every 6 weeks. According to the response evaluation criteria in solid tumor (RECIST) guidelines, we observed a stable disease. In parallel, the pain intensity progressively decreased and the asthenia became markedly less, although the site of the open biopsy has not healed and a daily and abundant necrotic material flow continues to be observed. Since January 2012, the patient was examined regularly. During the last visit, we observed the following: excellent general condition, absence of pain, remaining necrotic material including macroscopic calcifications flows (fig. 2) and calcification of the primary tumor (fig. 1b, fig. 3) and the metastasis (fig. 1d). Response and symptomatic improvement maintained until November 2013.

The patient had formally consented for the publication of the case report to be published.

\section{Conclusions}

To our knowledge, this is the first report of a MRCL treated with trabectedin that resulted in a substantial calcification of the primary tumor and metastases. After 14 cycles of trabectedin treatment, a durable partial response on metastases and stable disease on the primary tumor was accompanied with an improvement of symptoms and performance status, and with an acceptable toxicity profile. Therefore, this case emphasizes the role of trabectedin as a viable chemotherapeutic agent which significantly reinforces the therapeutic armamentarium in the treatment of MRCL.

In a former prospective multicenter phase II clinical trial, 3 of 23 assessable patients with myxoid liposarcoma, treated with neoadjuvant trabectedin, achieved a pathological complete response, i.e. a complete disappearance of the tumor tissue (histological and molecular absence of cells with the FUS-CHOP translocation) (13\%; 95\% CI: 3-34) and a good and a moderate histological response was observed in 2 and 10 patients, respectively, as assessed by a central pathological review. Furthermore, 7 out of 29 patients achieved a partial response according to RECIST (24\%; 95\% CI: 10-44) and 21 patients had a stable disease. Remarkably, no patients had a disease progression starting from the onset of the study treatment to the respective curative surgery. The high sensitivity of MRCL to the neoadjuvant trabectedin was in line with the findings of a retrospective analysis of 51 patients with MRCL, treated with trabectedin on a compassionate use protocol, where a high response rate (51\%) and a progression-free time (PFS) (14 months) were also reported [2]. Additionally, in a long-term follow-up in 32 of these 51 patients, the overall response rate was $50 \%$, with a PFS of 17 months, showing that that the high response rate of MRCL to trabectedin largely translates into a prolonged PFS [3].

In both abovementioned studies, substantial radiological changes in tumor density occurred, accompanied by histological decrement in the cellular and vascular tumor component and a maturation of tumor cells to lipoblasts in both myxoid and myxoid/round cell variants. Recently, it has been reported that the selective mechanism of trabectedin in MRCL is specific and related to its ability to cause a functional inactivation of the oncogenic chimera with a consequent depression of the adypocytic differentiation [1]. In vitro studies suggest that trabectedin induces the maturation of MRCL lipoblasts by inhibiting the fusion protein activity [5].

In conclusion, the rarity of response in this 63-year-old woman with MRCL was distinguished by the long-lasting ( $>27$ months) stable disease observed, even after drug discontinuation (15 months) and the presence of massive calcifications of the metastases and the 


\begin{tabular}{l|l}
\hline DOI: $10.1159 / 000360575$ & $\begin{array}{l}\text { C 2014 S. Karger AG, Basel } \\
\text { www.karger.com/cro }\end{array}$ \\
\hline
\end{tabular}

Turpin et al.: Tumor Calcification: A New Response Pattern of Myxoid Liposarcoma to Trabectedin

primary tumor. Physicians should be aware of this exceptional pattern of response in order to avoid inappropriate drug discontinuation.

\section{Acknowledgements}

The authors thank Y.-M. Robin, T. Ryckewaert and A. Tanović for their help with the writing and S. Marchant for the editing of this paper

\section{Disclosure Statement}

The authors declare no conflict of interest.

\section{References}

1 Di Giandomenico S, Frapolli R, Bello E, Uboldi S, Licandro SA, Marchini S, Beltrame L, Brich S, Mauro V, Tamborini E, Pilotti S, Casali PG, Grosso F, Sanfilippo R, Gronchi A, Mantovani R, Gatta R, Galmarini CM, Sousa-Faro JM, D'Incalci M: Mode of action of trabectedin in myxoid liposarcomas. Oncogene, 2013, Epub ahead of print.

-2 Grosso F, Jones RL, Demetri GD, Judson IR, Blay JY, Le Cesne A, Sanfilippo R, Casieri P, Collini P, Dileo P, Spreafico C, Stacchiotti S, Tamborini E, Tercero JC, Jimeno J, D'Incalci M, Gronchi A, Fletcher JA, Pilotti S, Casali PG: Efficacy of trabectedin (ecteinascidin-743) in advanced pretreated myxoid liposarcomas: a retrospective study. Lancet Oncol 2007;8:595-602.

-3 Grosso F, Sanfilippo R, Virdis E, Piovesan C, Collini P, Dileo P, Morosi C, Tercero JC, Jimeno J, D’Incalci M, Gronchi A, Pilotti S, Casali PG: Trabectedin in myxoid liposarcomas (MLS): a long-term analysis of a singleinstitution series. Ann Oncol 2009;20:1439-1444.

-4 Germano G, Frapolli R, Simone M, Tavecchio M, Erba E, Pesce S, Pasqualini F, Grosso F, Sanfilippo R, Casali PG, Gronchi A, Virdis E, Tarantino E, Pilotti S, Greco A, Nebuloni M, Galmarini CM, Tercero JC, Mantovani A, D'Incalci M, Allavena P: Antitumor and anti-inflammatory effects of trabectedin on human myxoid liposarcoma cells. Cancer Res 2010;70:2235-2244.

-5 Forni C, Minuzzo M, Virdis E, Tamborini E, Simone M, Tavecchio M, Erba E, Grosso F, Gronchi A, Aman P, Casali P, D'Incalci M, Pilotti S, Mantovani R: Trabectedin (ET-743) promotes differentiation in myxoid liposarcoma tumors. Mol Cancer Ther 2009;8:449-457.

-6 D'Incalci M, Galmarini CM: A review of trabectedin (ET-743): a unique mechanism of action. Mol Cancer Ther 2010;9:2157-2163.

7 Demetri GD, Chawla SP, von Mehren M, Ritch P, Baker LH, Blay JY, Hande KR, Keohan ML, Samuels BL, Schuetze S, Lebedinsky C, Elsayed YA, Izquierdo MA, Gómez J, Park YC, Le Cesne A: Efficacy and safety of trabectedin in patients with advanced or metastatic liposarcoma or leiomyosarcoma after failure of prior anthracyclines and ifosfamide: results of a randomized phase II study of two different schedules. J Clin Oncol 2009;27:4188-4196.

-8 Gronchi A, Bui BN, Bonvalot S, Pilotti S, Ferrari S, Hohenberger P, Hohl RJ, Demetri GD, Le Cesne A, Lardelli P, Pérez I, Nieto A, Tercero JC, Alfaro V, Tamborini E, Blay JY: Phase II clinical trial of neoadjuvant trabectedin in patients with advanced localized myxoid liposarcoma. Ann Oncol 2012;23:771-776. 
Turpin et al.: Tumor Calcification: A New Response Pattern of Myxoid Liposarcoma to Trabectedin
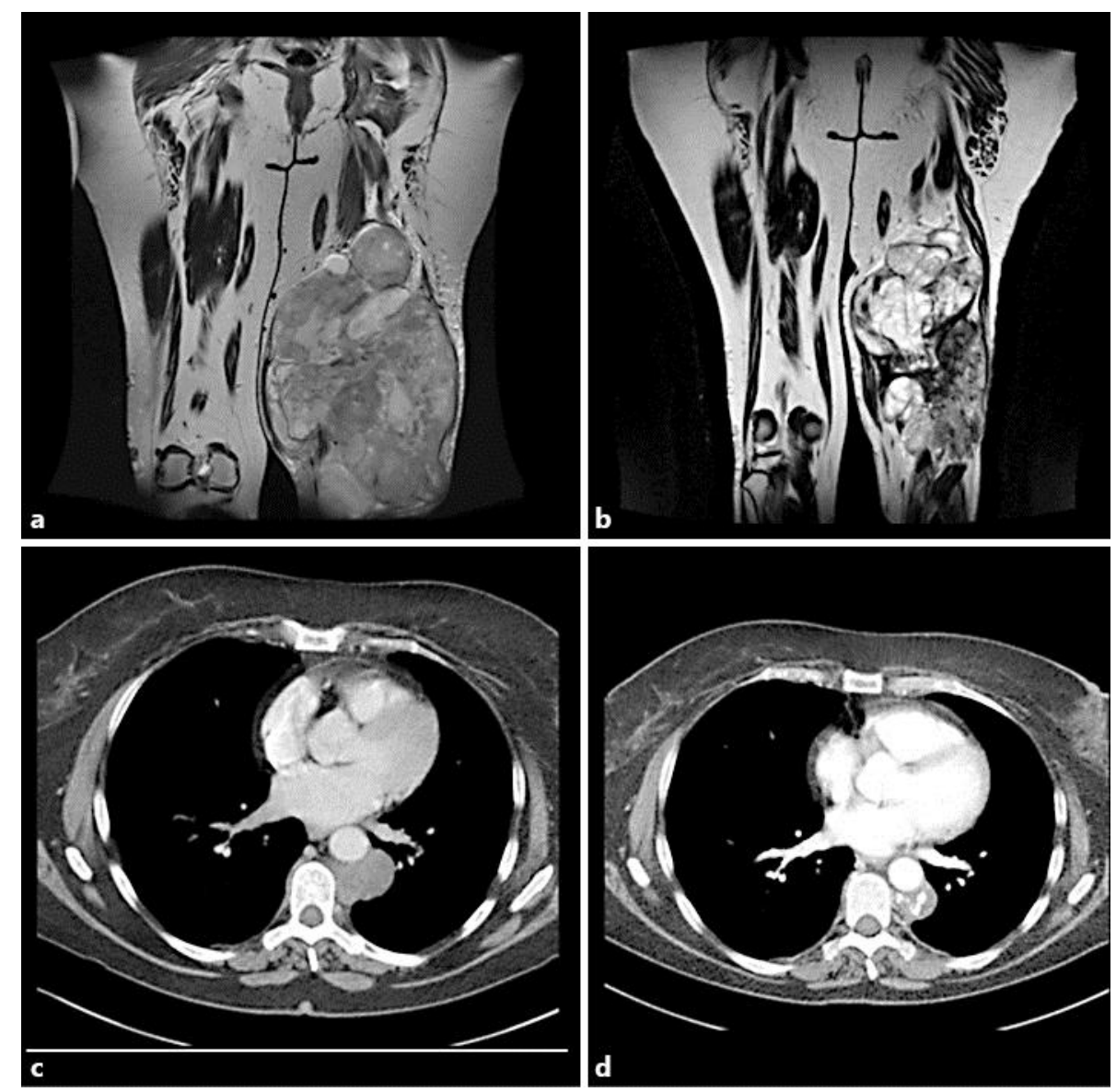

Fig. 1. Change in tumor pattern. MRI before chemotherapy (a), MRI after chemotherapy (b), CT scan before chemotherapy (c) and CT scan after chemotherapy (d).

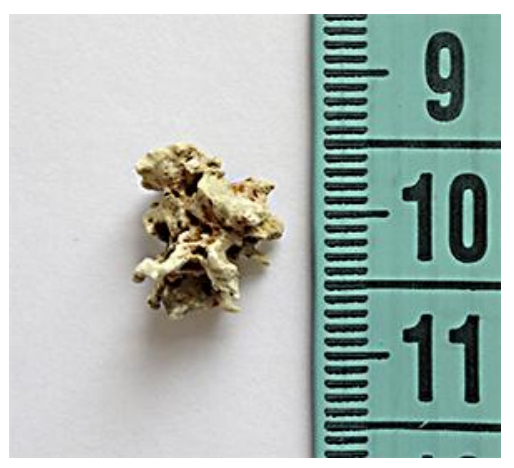

Fig. 2. Calcified material (15 mm) observed in the necrotic flow. 


\section{Case Reports in Oncology}

Case Rep Oncol 2014;7:204-209

DOI: 10.1159/000360575

Turpin et al.: Tumor Calcification: A New Response Pattern of Myxoid Liposarcoma to

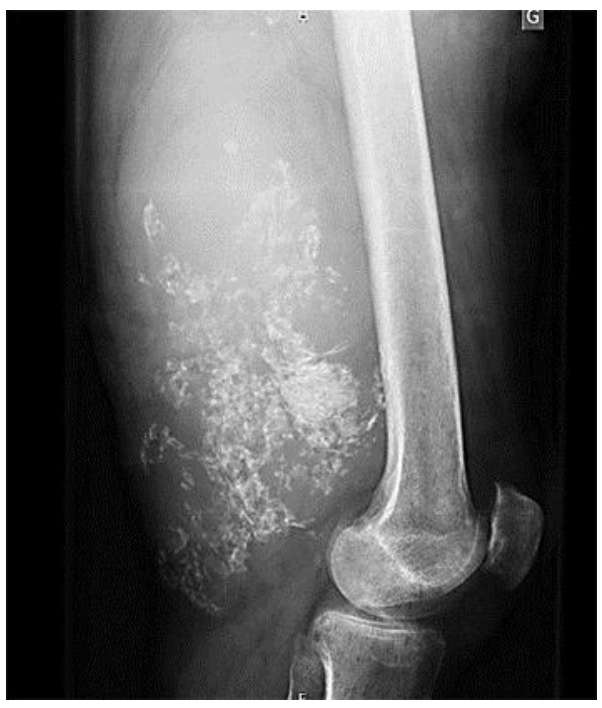

Fig. 3. Calcified primary tumor (standard X-ray). 